The effects of social anxiety on emotional face discrimination and its modulation by mouth salience

Andrew R. du Rocher and Alan D. Pickering.

Psychology Department, Goldsmiths, University of London, London, UK.

Andrew R. du Rocher (corresponding author), psp01adr@gold.ac.uk

Alan D. Pickering, a.pickering@ gold.ac.uk Tel: 02079197894 


\title{
The effects of social anxiety on emotional face discrimination and its modulation by mouth salience
}

\begin{abstract}
People high in social anxiety experience fear of social situations due to the likelihood of social evaluation. Whereas happy faces are generally processed very quickly, this effect is impaired by high social anxiety. Mouth regions are implicated during emotional face processing, therefore differences in mouth salience might affect how social anxiety relates to emotional face discrimination. We designed an emotional facial expression recognition task to reveal how varying levels of sub-clinical social anxiety (measured by questionnaire) related to the discrimination of happy and fearful faces, and of happy and angry faces. We also categorised the facial expressions by the salience of the mouth region (i.e., high [open mouth] vs. low [closed mouth]). In a sample of 90 participants higher social anxiety (relative to lower social anxiety) was associated with a reduced happy face reaction time advantage. However, this effect was mainly driven by the faces with less salient closed mouths. Our results are consistent with theories of anxiety that incorporate an oversensitive valence evaluation system.
\end{abstract}

Keywords: social anxiety; emotional faces; mouth salience.

\section{Introduction}

Social anxiety can be considered a continuum that ranges from people having no social worries at all, through to people experiencing sub-clinical levels of social anxiety, and in extreme cases manifesting as clinical social phobia (Rapee \& Heimberg, 1997). People high in social anxiety experience intense fear of social situations, due to the perceived risk of negative evaluation (Morrison \& Heimberg, 2013), and an increased 
fear of positive evaluation as this attracts attention from others (Weeks, Heimberg, Rodebaugh \& Norton, 2008). Cognitive-motivational models of anxiety suggest that those high in anxiety have an oversensitive valence evaluation system that results in a threat-related attentional bias (Mogg \& Bradley, 1998). The interaction between cognition, emotion, and anxiety is often studied experimentally using emotional facial stimuli as, in real life, recognising a person's facial expression aids the observer to identify a person's intentions and mood (Bruce \& Young, 1986).

Generally happy faces are recognised faster than negatively-valenced faces (Calvo \& Beltran, 2014; Leppanen \& Hietanen, 2004). However, higher sub-clinical social anxiety (relative to lower social anxiety) relates to slower reaction times (RTs) to happy faces (Silvia, Allan, Beauchamp, Maschauer \& Workman, 2006). Silvia et al. proposed that positive information concerning other people may be less readily available in high social anxiety, which may slow down associating conceptual information with the percept of a happy face.

Higher sub-clinical social anxiety has been related to an increased ability to detect briefly presented fearful faces that were immediately masked by neutral faces (Doty, Japee, Ingvar \& Ungerleider, 2013). Higher trait anxiety scores in a combined sample of social anxiety patients, generalised anxiety patients, panic disorder patients, and normal controls predicted greater changes in brain activity during fearful face processing relative to happy face processing, and also during angry face processing relative to happy face processing (Fonzo et al., 2015). Rapid responses were not a requirement of this emotional face-matching task, and thus no anxiety-related RT effects were observed. However, research using an ambiguous expression recognition task suggests that social anxiety disorder patients identify faces as being angry more quickly than they identify faces as being happy (Maoz et al., 2016). This effect was not present in non- 
anxious controls. These studies suggest that social anxiety might relate to faster RTs for angry and fearful faces relative to happy faces, but the literature on social anxiety and emotional face recognition needs further development.

Mouth regions have been shown to be highly implicated during emotional face processing (Calvo \& Beltran, 2014; Calvo \& Nummenmaa, 2008). The display of teeth (relative to no display of teeth) increases valence and arousal ratings for happy faces and grimacing faces (daSilva et al., 2016). Accordingly, one might expect happy, fearful, and angry facial expressions with salient open mouths, to facilitate an information processing advantage relative to those with less-salient closed mouths. This might modulate how socially anxious people process emotional faces.

This purpose of this experiment is to reveal how social anxiety relates to emotional face processing in two specific situations (i.e., discriminating between happy and fearful faces, and between happy and angry faces). Although a person expressing fear might initiate avoidance behaviour, a person perceiving fear might initiate approach behaviour in order to help the fearful person. Moreover, although a person expressing anger might initiate approach behaviour, a person perceiving anger might initiate avoidance behaviour (Marsh, Ambady \& Kleck, 2005). Therefore although both fearful and angry faces signal a potential social threat in the environment, they may elicit different social responses. Considering that these social responses may differ, it is important to determine whether social anxiety still predicts similar effects upon RTs when discriminating between fearful and happy faces, compared to when discriminating between angry and happy faces.

We predict in general that happy faces will be responded to faster than threatrelated (fearful or angry) faces, but based upon Silvia et al. (2006) we predict that this happy face RT advantage will be reduced in high social anxiety. Moreover, based upon 
Doty et al. (2013), and the theory that anxiety relates to enhanced threat processing (Mogg \& Bradley, 1998), we predict that social anxiety will relate to faster RTs for threat-related faces. However, our factorial design will also test whether social anxiety has a different effect when discriminating between happy and fearful faces than it does when discriminating between happy and angry faces. We will also determine how the saliency of the mouth regions of the faces modulate any social anxiety effects that are present.

\section{Method}

\section{Participants}

We recruited participants with no reported history of neurological disorder $(\mathrm{N}=90,64$ female) from Goldsmiths, University of London, with a mean age of $24.6(\mathrm{SD}=6) .76$ of these participants were right handed. 22 participants were $1^{\text {st }}$ year psychology undergraduates who participated in return for course credit. The rest were paid $£ 10$ and were students and staff from other departments. This study was approved by the Goldsmiths psychology departments' ethics committee (approval received 24/10/2012). Participants gave informed written consent consistent with standard ethical practices. Our sample size was chosen to facilitate $80 \%$ power for two-tailed tests at $\mathrm{p}=0.05$, for a correlation of 0.3 , which is typical for personality-behaviour associations. 8 participants were excluded from the analyses due to data saving problems.

\section{Psychometric measures}

Social anxiety was assessed using the Social Phobia Scale (SPS) and the Social Interaction Anxiety Scale (SIAS) developed by Mattick and Clarke (1998). Consistent 
with previous research (Silvia et al., 2006) we standardised and averaged the total scores of the SIAS and total scores of the SPS to obtain a unitary social anxiety score. Trait anxiety was assessed with the trait scale of the State-Trait Anxiety Inventory (STAI, Spielberger et al., 1983).

\section{Stimuli}

The emotional faces used were obtained from a standardised face stimuli set developed for research (NimStim; Tottenham et al., 2009). The individual face pictures were $60 \mathrm{~mm}$ high and $48 \mathrm{~mm}$ wide when presented on a 15.5 inch laptop screen. Three face stimulus sets containing different people's faces in each were created for use in two tasks. Each of the three face sets included happy, fearful and angry facial expressions (with equal proportions of open mouths and closed mouths) posed by six different models from the NimStim (see supplementary materials for examples). Thus, in each task, 24 unique stimuli were presented ( 2 emotions x 2 mouth types x 6 people).

\section{Procedure}

Participants were told that they would be presented with two short emotional facial expression recognition tasks (i.e., conditions) with a short rest in between. Participants were asked to sit as close to the screen as was comfortable for their eyes (typical viewing distance was approximately $70 \mathrm{~cm}$ ). The task instructions were presented on the screen. To start each task the first screen instructed participants that they would have to identify the emotional expression shown in photographs of faces (happy and fear in the one task; and happy and anger in the other task). Participants were then shown examples of the various faces they might see. They were told to rest their index fingers over the response keys ( $\mathrm{z}$ and /) and to respond as fast as possible while maintaining 
high accuracy levels. They were verbally told that a (quiet) high pitched tone following a response indicates a correct response, whereas a (quiet) low pitched tone following a response indicates an incorrect response (this ensured participants were sure of the response mappings).

The experimental stimuli were displayed until a response key was pressed. Unbeknown to the participants, at the beginning of each task, there were 24 trials included as practice trials; these were discarded and not analysed. The main experimental stimuli that followed consisted of 120 happy face trials and 120 threatrelated face trials (randomly intermixed in each of the two tasks). The emotional facial expression discrimination paradigm was designed primarily to elicit RT effects as opposed to errors. The trial type sequence was created using a random number generator. It was the same for all participants as this is an individual differences study, and we wanted as few uncontrolled variables as possible to vary across participants. We also used the same trial type sequence for each of the tasks (with fearful faces being substituted with angry faces where appropriate). There were 240 non-practice trials in total in each task so we felt that there was no chance that using the same sequence in each task would cause any learning of the sequence of trial types and, as noted below, we counterbalanced the order of the 2 tasks across participants. Each task lasted for approximately ten minutes. The experiment was thus created using a 2 X 2 X 2 combination of valence (happy face versus threat-related face) x mouth type (closed mouth versus open mouth) $\mathrm{x}$ discrimination task (happy/fear discrimination versus happy/anger discrimination) repeated-measures design. We included equal proportions of male and female faces, with different facial identities used across the two tasks. The left/right finger response key mappings were also counterbalanced. Each participant was tested using a different one of three face identity sets for each of the two tasks (i.e., a 
different face set was used per task for each participant, and the mapping of the face-set was counterbalanced using an incomplete Latin square).

\section{Data analysis}

Our analysis comprises five parts. RT outliers for correct responses were removed if RTs $<200$ msecs and RTs $>1250$ msecs ( $4.3 \%$ of correct responses were excluded). First the RT data for correct responses were subjected to a 2 (valence; happy face versus threat-related face) x 2 (mouth type; salient open mouth versus less-salient closed mouth) x 2 (discrimination task; happy/fear discrimination versus happy/anger discrimination) repeated-measures ANCOVA, with the unitary social anxiety scores as the covariate.

The above main effect of valence thus tests our prediction that happy faces will be responded to faster than threat-related faces. The social anxiety effects appear initially as the covariate interactions with the above effects of valence, mouth type, and discrimination task. These covariate interactions test whether social anxiety modulates the RT difference between happy faces and threat-related faces, the RT difference between open and closed mouthed faces, and/or the RT difference between the happy/fear and happy/anger tasks, respectively. The more complex interactions will test whether social anxiety modulates any interactions between the 3 repeated-measures factors. For example, the valence by discrimination task by social anxiety interaction tests whether social anxiety affects discriminating between happy and fearful faces differently from discriminating between happy and angry faces. We also report the equivalent bivariate correlation corresponding to the covariate interaction that shows the relationship between social anxiety and the difference in RTs for threat-related and happy faces. This critical covariate interaction (correlation) tests our main hypothesis that the happy face RT advantage will be reduced in high social anxiety. 
The second part of our analysis focused upon conducting planned one-way ANCOVAs and/or bivariate correlations to explain the resulting interactions present in the above analysis. In short, this analysis separates out how social anxiety affects RTs for the open-mouthed and closed-mouthed happy and threat-related faces.

The third part of our analysis evaluated two key social anxiety correlations present in the above analysis, this time using partial correlation to control for the general form of trait anxiety that is measured by participants' STAI scores.

The fourth part of our analysis tests whether social anxiety was correlated with RTs to any of the individual trial types. Here we test our hypotheses that social anxiety will relate to faster RTs to threat-related faces, and test whether the correlations are affected by the salience of the mouth regions of the emotional faces. However, here it is important to control for general RT effects. General RT effects are subtracted out in the RT difference computations (trial type RT differences), but are present in the individual trial type RTs. Thus, if general RTs are a major source of variance in every condition, and are not related to social anxiety, then this will act as error variance and so suppress the study's ability to detect anxiety relationships with RTs to individual trial types. Thus, we calculated a general RT factor using exploratory factor analysis (the details of this are contained in our supplementary materials). We then controlled for this general RT factor when running exploratory correlations between social anxiety and the key individual trial types.

The fifth and sixth parts of our analysis concerned confirming that gender differences and the effect of time on task did not adversely affect the interpretation of the above analyses. Gender differences were examined using t-tests and correlations. The effect of time on task was examined using a robust generalised linear model. These $5^{\text {th }}$ and $6^{\text {th }}$ analyses are presented in our supplementary materials. 


\section{Results}

\section{Psychometric measurement}

Participants SIAS scores ranged from 0-70 $($ mean $=21.2, \mathrm{SD}=12.0)$, and their SPS scores ranged from 3-60 $($ mean $=17.4, \mathrm{SD}=11.8)$. Figure $1($ panel B $)$ shows the distribution of the standardised and averaged scores of the SIAS and SPS (i.e., their unitary social anxiety scores). Participants STAI trait scale scores ranged from 23-69 $($ mean $=42.6, \mathrm{SD}=10.0)$.

\section{Reaction times}

The detailed descriptive statistics for the RTs for each of the eight trial types are presented in our supplementary materials. The main effect of valence was significant $\left(F[1,80]=7.5, p=0.008, \eta^{2}=0.086\right)$, and this also significantly interacted with social anxiety $\left(F[1,80]=6.4, p=0.013, \eta^{2}=0.074\right)$. Therefore, there was a small but significant difference between average RTs for happy faces from both discrimination tasks (645 msecs; SE 10), and average RTs for threat-related faces from both discrimination tasks (652 msecs; SE 10). However, even though this effect was quite small the interaction shows that social anxiety correlated significantly with the RT difference (mean RT for threat-related faces minus mean RT for happy faces). The correlation value was $r=-0.27$. This correlation is depicted in Figure 1 (panel C), which shows that higher social anxiety was related to a reduced happy face RT advantage (threat-related face RT minus happy face RT).

The main effect of mouth type was also significant $(F[1,80]=91.9, p<0.001$, $\left.\eta^{2}=0.535\right)$. This shows that facial expressions with open mouths were on average 
responded to significantly faster (639 msecs; SE 10) than facial expressions with closed mouths (658 msecs; SE 10). Mouth type did not significantly interact with social anxiety $\left(F[1,80]=1.3, p=0.254, \eta^{2}=0.016\right)$.

The main effect of discrimination task was not significant $(F[1,80]=0.1, p=0.722$, $\left.\eta^{2}=0.002\right)$, and did not significantly interact with social anxiety $(F[1,80]=0.4, p=0.534$, $\left.\eta^{2}=0.005\right)$. However, the interaction between valence and mouth type was significant $\left(F[1,80]=13.1, p=0.001, \eta^{2}=0.141\right)$, but this interaction did not significantly interact with social anxiety $\left(F[1,80]=0.3, p=0.587, \eta^{2}=0.004\right)$. Figure 1 (panel A) suggests that the RT advantage for the more salient open mouths, relative to closed mouths, was reduced when processing threat-related faces relative to happy faces. We return to this valence $\mathrm{x}$ mouth type interaction for further statistical analyses below. The interaction between valence and task, the interaction between mouth type and discrimination task, the 3-way interaction between valence, mouth type and discrimination task, and their interactions with social anxiety were all non-significant (all $F \mathrm{~s}<1.2$, all $p \mathrm{~s}>0.3$ ).

Figure 1 (panel A) illustrates the interaction between valence and mouth type reported above. Our planned analysis of this interaction required an adjusted significance level of $0.05 / 4$ for each of the following four one-way ANCOVAS. Happy faces with open mouths were responded to faster (632 msecs; SE 11) than happy faces with closed mouths (659 msecs; SE 10). This RT advantage was highly significant $\left(F[1,80]=100.9, p<0.001, \eta^{2}=0.558\right)$. Threat-related faces with open mouths were responded to faster (646 msecs; SE 11) than threat-related faces with closed mouths (657 msecs; SE 10). This RT advantage was significant $(F[1,80]=11.3, p=0.001$, $\left.\eta^{2}=0.124\right)$. Neither of these two comparisons significantly interacted with social anxiety (both $F \mathrm{~s}<1.3$, both $p \mathrm{~s}>0.20$ ). 


\section{Insert Figure 1}

Threat-related faces with open mouths were responded to slower than happy faces with open mouths. This RT effect was significant $\left(F[1,80]=14.9, p<0.001, \eta^{2}=0.157\right)$. This comparison did not significantly interact with social anxiety $(F[1,80]=1.7$, $\left.p=0.193, \eta^{2}=0.021\right)$. However, RTs for threat-related faces with closed mouths were not significantly different from RTs for happy faces with closed mouths $(F[1,80]=0.2$, $\left.p=0.643, \eta^{2}=0.003\right)$. Interestingly, this non-significant effect interacted robustly with social anxiety $\left(F[1,80]=7.6, p=0.007, \eta^{2}=0.087\right)$. This interaction with social anxiety tests the correlation between social anxiety and the RT difference between threat-related faces with less salient closed mouths and happy faces with less salient closed mouths. The correlation value was $r=-0.30$. As participants were more socially anxious their RTs to happy faces were less rapid (relative to their RTs for threat-related faces), in the condition using closed-mouth face stimuli.

The last two one-way ANCOVAs illustrate how the reduced happy face RT advantage in high social anxiety may be driven somewhat more by the closed mouth trials, than the open mouth trials. However, it should be remembered that the formal social anxiety $\mathrm{x}$ valence $\mathrm{x}$ mouth type interaction was not significant, as noted above. We also confirmed that the correlation between social anxiety and the RT difference between threat-related faces and happy faces (across both mouth conditions combined), and the correlation between social anxiety the RT difference between closed mouthed threat-related faces and closed mouthed happy faces, both remained significant when controlling for trait anxiety (both $r s=-0.26$, both $p s=0.018$ ).

The exploratory zero-order correlations in Table 1 suggested that social anxiety was not correlated with RTs to any of the individual trial types. However, Table 1 also 
shows that when controlling for the general RT factor using exploratory partial correlation, social anxiety displayed a negative correlation with RTs to threat-related faces with closed mouths, and a positive correlation with RTs to happy faces with closed mouths. That is to say, the above effects of social anxiety upon the RT differences between happy and threat-related faces appear to be being driven mainly by faster RTs to threat-related faces with less-salient closed mouths, and to a lesser extent slower RTs to happy faces with less-salient closed mouths. However, these exploratory correlations were not adjusted for multiple testing and should be interpreted with caution.

\section{Insert Table 1}

A robust generalised linear model was used to explore the effects of time on task on the above results (see supplementary materials for details). In sum, this variable had no significant effect on the above findings.

\section{Discussion}

We found that RTs to happy faces were modestly but significantly faster than RTs to threat-related faces. This is consistent with the finding that happy faces are recognised faster than negatively-valenced faces (Calvo \& Beltran, 2014; Leppanen \& Hietanen, 2004). However, Figure 1 (panel C) shows that social anxiety was significantly related to a reduced happy face RT advantage, which is consistent with the study by Silvia et al. (2006). Importantly, there were no RT effects, or social anxiety effects, that differed between fearful and angry face trials. Our supplementary materials also show that emotional face recognition accuracy was unaffected by social anxiety. 
Facial expressions with salient open mouths were responded to faster than facial expressions with less salient closed mouths. However, Figure 1 (panel A) shows that the RT advantage for the more salient open-mouthed faces, relative to closed-mouth faces, was reduced when processing threat-related faces relative to happy faces. This suggests that the salience of the mouth region enhances emotional expression recognition more during the processing of happy faces, which is consistent with the study by Calvo and Beltran (2014).

Although threat-related faces with open mouths were responded to slower than happy faces with open mouths, mean RTs for threat-related faces with closed mouths were similar to mean RTs for happy faces with closed mouths. There was a reduced happy face RT advantage in high social anxiety across the whole study, but the effect appeared to be driven mainly by responses to the happy and threat-related faces with closed mouths (and not by the stimuli with open mouths). However, the 3-way interaction effect relating to this difference was not significant, and so this effect should be interpreted with caution.

Critically, we showed that the correlations between social anxiety and the reduced happy face RT advantage remained after controlling for a general form of trait anxiety measured using the STAI. Thus, the effects seem to be specific to social anxiety per se. Moreover, Table 1 shows that when controlling for general sources of RT variance, social anxiety had a negative correlation with RTs to threat-related faces and a positive correlation with RTs to happy faces. Again, these effects were more marked for the closed-mouthed faces.

Our results suggest that the processing of emotional faces with salient open mouths was similar at all levels of social anxiety. However, the threat-related faces with lesssalient closed mouths appeared to be processed as being threat-related faster by those 
high in social anxiety, relative to those low in social anxiety. This finding lends some support to the cognitive motivational account of anxiety proposed by Mogg and Bradley (1998). Mogg and Bradley suggest that stimuli indicating a mild level of threat will be processed as being more threat-relevant by those high in anxiety relative to those low in anxiety, as high anxiety is related to an oversensitive valence evaluation system.

Silvia et al. (2006) proposed that the reduced happy face RT advantage in social anxiety might reflect the reduced availability of positive information concerning other people slowing down associating conceptual information with the percept of a happy face. However, an alternative explanation can be offered. Psychophysiological evidence suggests that perception of the mouth region of happy faces occurs very quickly, and that the resulting information facilitates a cognitive shortcut aiding the identification of the happy expression (Calvo and Beltran, 2014). This could account for the happy face $\mathrm{RT}$ advantage. It is possible that this cognitive shortcut is less effective and/or deployed less in high social anxiety, and instead attentional resources are preferentially directed towards sources of perceptual information that may be indicative of a threat-related face. This effect may be particularly evident when the perceived faces have less salient closed mouths, as opposed to salient open mouths. Interestingly, this differential emotional face processing effect of high social anxiety may function independently of general trait anxiety.

We close by discussing some limitations to this work. Firstly, female participants outnumbered male participants. However, our supplementary materials show that there were no gender differences in RTs to the emotional faces, and the correlations between social anxiety and the key RT effects were only slightly stronger when just the females were analysed (relative to when the whole sample was analysed). Thus, we are not overly concerned with this issue. Secondly, our high social anxiety participants were not 
clinical patients. We can only speculate that the emotional processing effects that related to high social anxiety in our study would generalise to those with clinical social phobia. Thirdly, happy faces appeared in both discrimination tasks (although the person identities were different) and so participants practice on twice as many happy faces as fear or angry faces. However, we do not expect this to have facilitated any learning effect, as the happy face RT advantage was quite small. Fourthly, we used response feedback tones which were quiet, however we cannot be definitely sure that this did not increase performance anxiety. Future research should test whether our results are replicated in situations with and without the presence of feedback tones. Fifthly, although we controlled for general trait anxiety, we did not control for general state anxiety. Future research should test whether the social anxiety effects upon face processing are increased by general state anxiety.

In conclusion, high social anxiety was related to a reduced happy face RT advantage. We suggest that this was due to a processing bias for threat-related faces, relative to happy faces. However, this effect was only reliable when the facial expressions are shown with less salient closed mouths. Stimuli indicating a mild level of social threat appeared to be processed as being more threat-relevant by those high in social anxiety, relative to those low in social anxiety. This is consistent with the account of anxiety proposed by Mogg and Bradley (1998), which suggests that high anxiety is associated with an oversensitive valence evaluation system. 


\section{Acknowledgment}

This work was supported by a doctoral studentship awarded to the first author by

Goldsmiths psychology department.

\section{Conflicts of interest}

The authors have no conflicts of interest to declare. 


\section{References}

Bruce, V., \& Young, A. (1986). Understanding face recognition. British Journal of Psychology, 77, 305-327. DOI:10.1111/j.2044-8295.1986.tb02199.x

Calvo, M. G. \& Beltran, D. (2014). Brain lateralization of holistic versus analytic processing of emotional facial expressions. Neuroimage, 92, 237-247. DOI:10.1016/j.neuroimage.2014.01.048

Calvo, M. G. \& Nummenmaa, L. (2008). Detection of emotional faces: Salient physical features guide effective visual search. Journal of Experimental Psychology: General, 137, 3, 471-494. DOI:10.1037/a0012771

daSilva, E. B., Crager, K., Geisler, D., Newbern, P., Orem, B., \& Puce, A. (2016). Something to sink your teeth into: The presence of teeth augments ERPs to mouth expressions. Neuroimage, 127, 227-241. DOI:10.1016/j.neuroimage.2015.12.020

Doty, T. J., Japee. S., Ingvar, M., \& Ungerleider, L. G. (2013). Fearful face detection sensitivity in healthy adults correlates with anxiety-related traits. Emotion, 13, 2, 183188. DOI:10.1037/a0031373

Fonzo, G. A., Ramsawh, H. J., Flagan, T. M., Sullivan, S. G., Letamendi, A., Simmons, A. N., Paulus, M. P., \& Stein, M. B. (2015). Common and disorder-specific neural responses to emotional faces in generalised anxiety, social anxiety and panic disorders. 
The British Journal of Psychiatry: The Journal of Mental Science. January 2015, 1-10. DOI:10.1192/bjp.bp.114.149880

Leppanen, J. M. \& Hietanen, J, K. (2004). Positive facial expressions are recognised faster than negative facial expressions, but why? Psychological Research, 69, 22-29. DOI:10.1007/s00426-003-0157-2

Maoz, K., Eldar, S. Stoddard, J., Pine, D. S., Leibenluft, E. \& Bar-Haim, Y. (2016). Angry-happy interpretations of ambiguous faces in social anxiety disorder. Psychiatry Research, 241, 122-127. DOI:10.1016/j.psychres.2016.04.100

Marsh, A. A., Ambady, N., \& Kleck, R. E. (2005). The effects of fear and anger facial expressions on approach and avoidance related behaviours. Emotion, 5 (1), 119-124. DOI:10.1037/1528-3542.5.1.119

Mattick, R. P \& Clarke, J, C. (1998). Development and validation of measures of social phobia scrutiny fear and social interaction anxiety. Behaviour Research and Therapy, 36, 455-470. DOI:10.1016/S0005-7967(97)10031-6

Mogg, K., \& Bradley, B. P. (1998). A cognitive-motivational analysis of anxiety. Behaviour Research and Therapy, 36, 809-848. DOI:10.1016/S0005-7967(98)00063-1

Morrison, A. S., \& Heimberg, R. G. (2013). Social anxiety and social anxiety disorder. Annual Review of Clinical Psychology, 9, 249-274. DOI:10.1146/annurev-clinpsy050212-185631 
Rapee, R. M., \& Heimberg, R. G. (1997). A cognitive-behavioural model of anxiety in social phobia. Behaviour Research and Therapy, 35, 741-756. DOI:10.1016/S00057967(97)00022-3

Silvia, P. J., Allan, W. D., Beauchamp, D. L., Maschauer, E. L., \& Workman, J. O. (2006). Biased recognition of happy facial expressions in social anxiety. Journal of Social and Clinical Psychology, 25, 585-602.

Spielberger, C. D., Gorsuch, R. L., Lushene, R., Vagg, P. R., \& Jacobs,G. A. (1983). Manual for the State-Trait Anxiety Inventory. Palo Alto, CA: Consulting Psychologists Press.

Tottenham, N., Tanaka, J. W., Leon, A. C., McCarry, T., Nurse, M., Hare, T. A., et al. (2009). The NimStim set of facial expressions: judgments from untrained research participants. Psychiatry Research. 168, 242-249. DOI:10.1016/j.psychres.2008.05.006

Weeks, J. W., Heimberg, R. G., Rodebaugh, T. L., \& Norton, P. J. (2008). Exploring the relationship between fear of positive evaluation and social anxiety. Journal of Anxiety Disorders, 22, 386-400. DOI:10.1016/j.janxdis.2007.04.009 

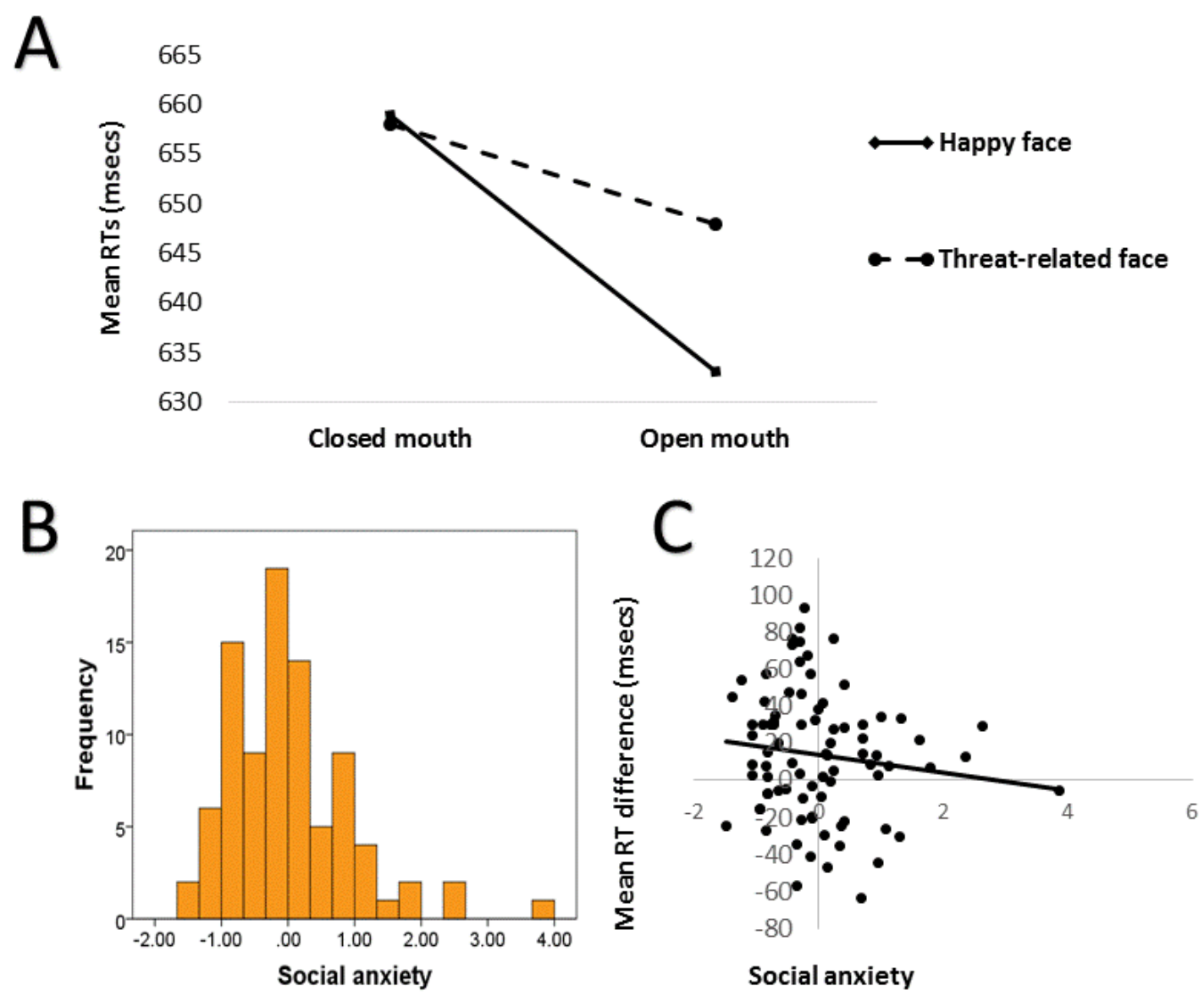

Figure 1: Panel A shows the RT interaction between the valence of the emotional faces (happy versus threat-related) and mouth type (open versus closed). Panel B shows the distribution of the social anxiety scores (i.e., the standardised and averaged scores of the SIAS and the SPS). Panel C shows the negative correlation between these social anxiety scores and the overall happy face RT advantage. 
Table 1: The zero correlations between social anxiety and the key trial types, and the partial correlations between social anxiety and the key trial types (controlling for general RT variance).

\begin{tabular}{lllll}
\hline & \multicolumn{2}{l}{ Zero-order } & \multicolumn{2}{l}{ Partial } \\
& $r$ & $p$ & $r$ & $p$ \\
Threat-related (averaged across & -0.09 & 0.42 & -0.25 & 0.03 \\
mouth types) & & & & \\
Happy (averaged across mouth & -0.03 & 0.83 & 0.28 & 0.01 \\
types) & & & & \\
Threat-related closed mouth & -0.11 & 0.34 & -0.30 & 0.008 \\
Happy closed mouth & -0.03 & 0.79 & 0.22 & 0.05 \\
Threat-related open mouth & -0.07 & 0.52 & -0.07 & 0.53 \\
Happy open mouth & -0.02 & 0.87 & 0.18 & 0.11 \\
\hline
\end{tabular}

